Supporting Information (SI) for

\title{
Quantitative Description of Catalysis of Inherent Metallic Species in Lignite Char during $\mathrm{CO}_{2}$ Gasification
}

Nurulhuda Halim, U.P.M. Ashik, Xiangpeng Gao, Shinji Kudo, Edy Sanwani, Koyo Norinaga, and Jun-ichiro Hayashi 


\section{SI.1. SEM observation of particles of A0 lignite after ball milling}
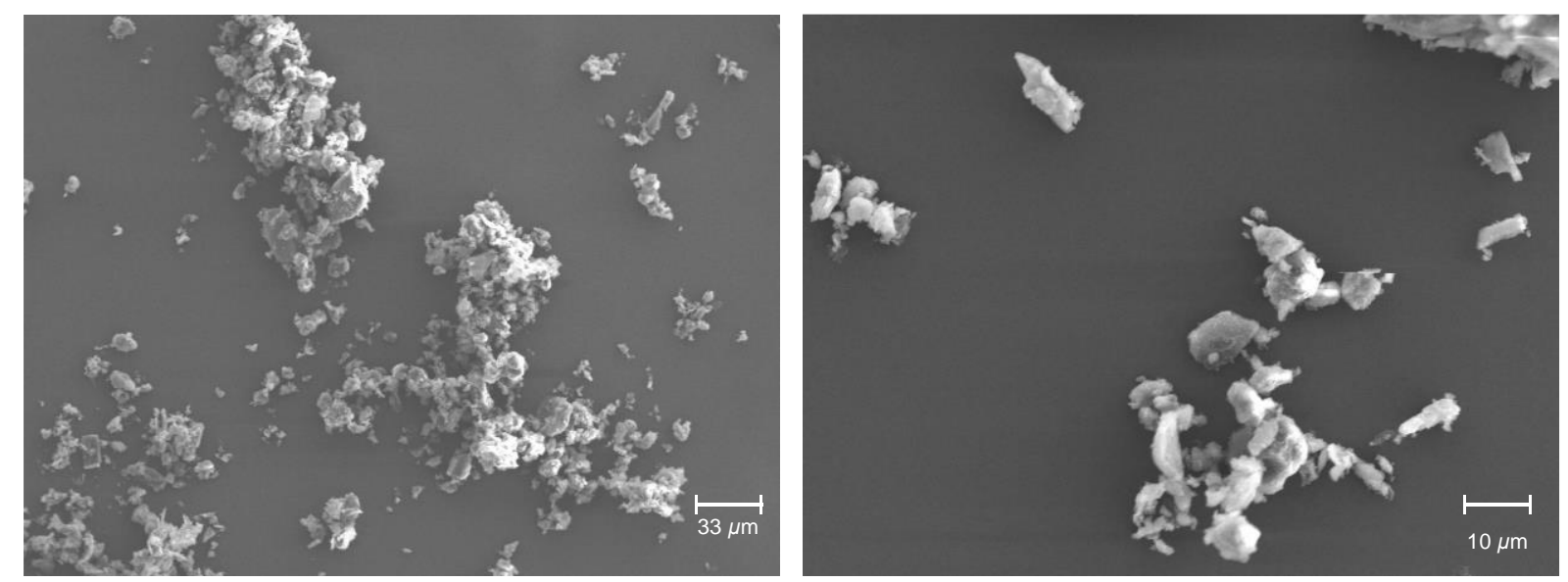

Figure SI.1. SEM images of lignite particles of A0 lignite after pulverization by ball milling for $10 \mathrm{~h}$. The particles sizes were reduced to $<10 \mu \mathrm{m}$. 


\section{SI.2. Flow diagram of sequential leaching of metallic species}

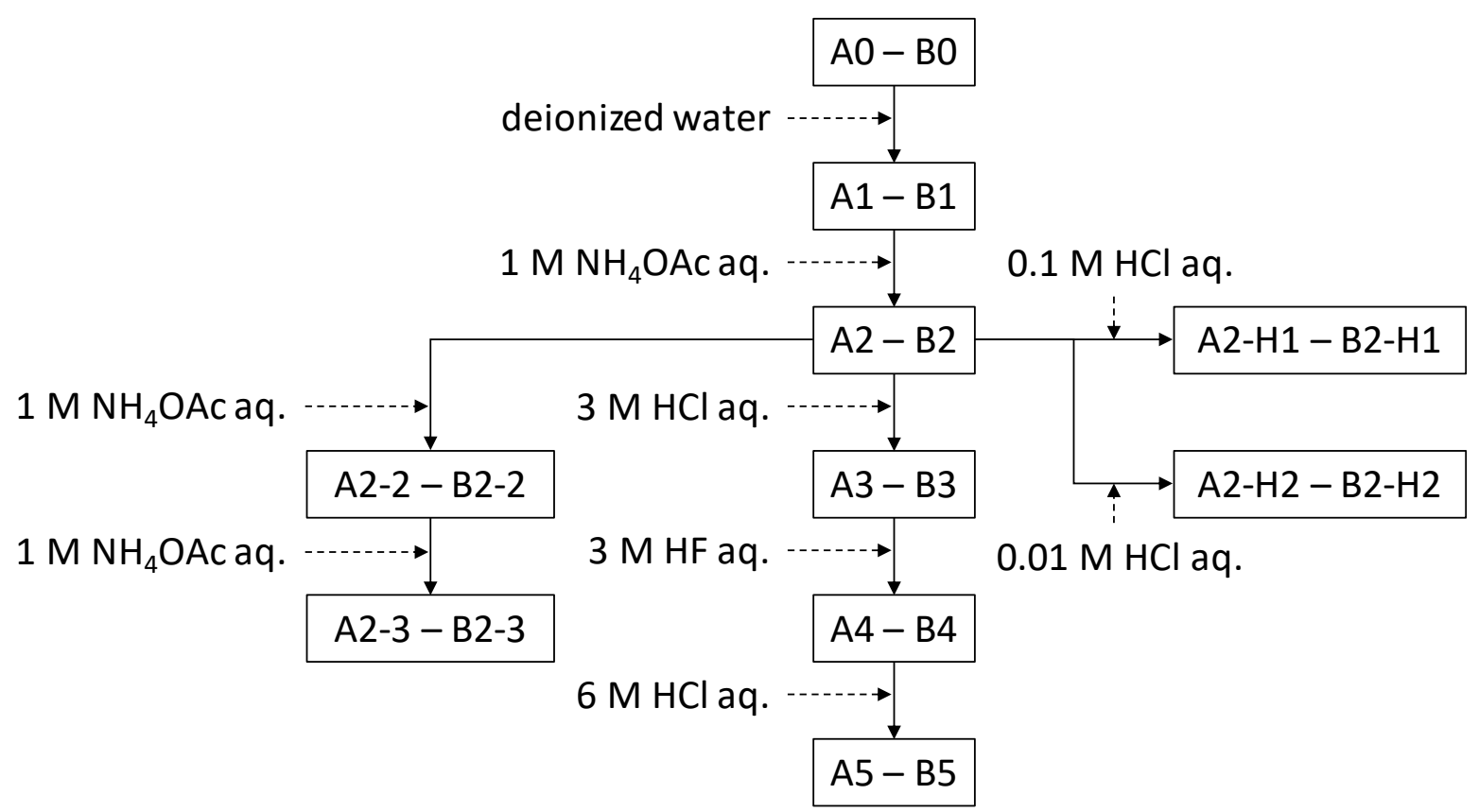

Figure SI.2. Flow diagram of sequential washing. The washing of $A 1$ and that of $B 1$ with $1 M$ $\mathrm{NH}_{4} \mathrm{OAc}$ aq. were repeated three times, respectively, and thereby A2-3, A2-3, B2-2 and B2-3 were obtained. Such repeated washing was performed to remove ion-exchanged metallic species. As a result, it was suspected that substantial portions of the ion-exchanged metallic species were left in the lignites even after the repeated washing. A2 and B2 were also washed with $0.01 \mathrm{M} \mathrm{HCl}$ aq. ( $\mathrm{pH} \approx 2$ ) or $0.1 \mathrm{M} \mathrm{HCl}$ aq. ( $\mathrm{pH} \approx 1)$ to investigate the effect of $\mathrm{pH}$ of acidic solution on the removal of metallic species. 


\section{SI.3. Effect of initial char mass on the $\mathrm{d} X / \mathrm{d} t$ profiles of $\mathrm{CO}_{2}$ gasification of chars from A0 and BO}
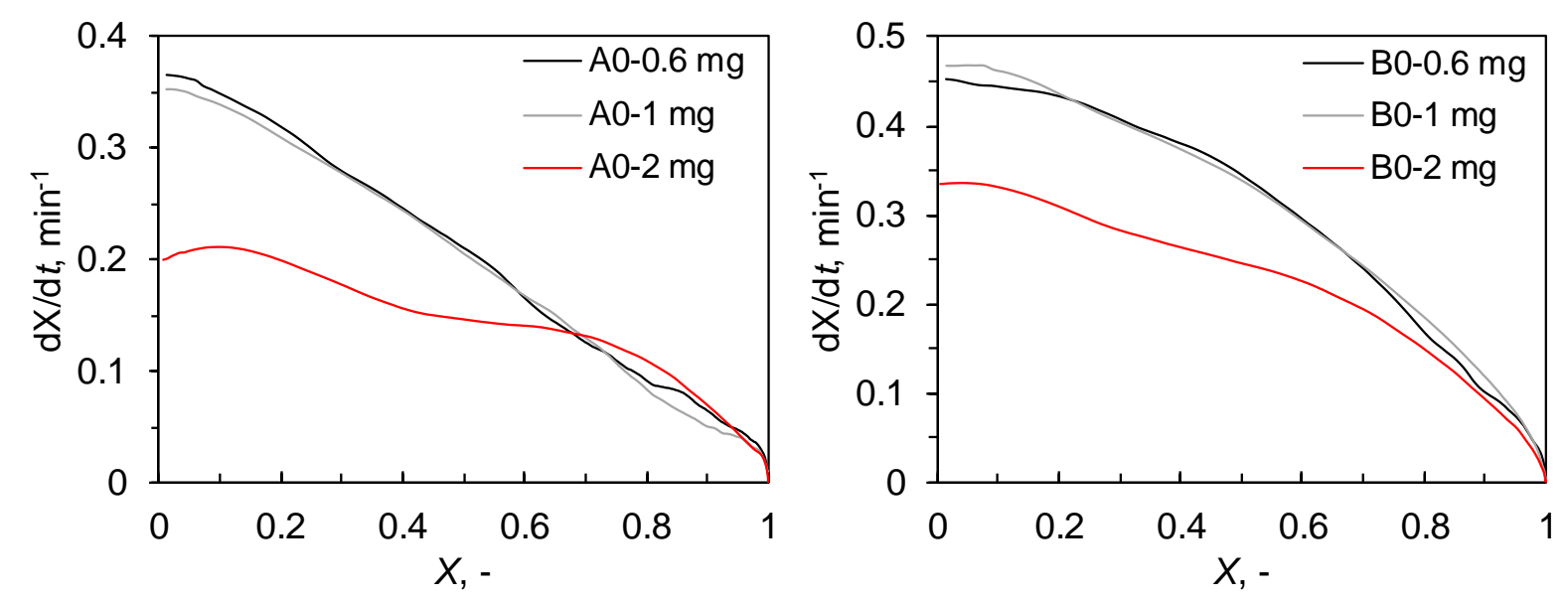

Figure SI.3. Measured changes in $\mathrm{d} X / \mathrm{d} t$ with $X$ for gasification of $\mathrm{A} 0$ and $\mathrm{B} 0$ chars with initial char mass of $0.6,1$ or $2 \mathrm{mg}$. Each graph shows that the initial mass of $1.0 \mathrm{mg}$ is sufficiently small at which the rate of gasification is chemically controlled without signicant resistances to mass transport within the char bed. 


\section{SI.4. Details of kinetic model}

The following kinetic equation, eq.I, describes the overall conversion rate of char, $\mathrm{d} X / \mathrm{d} t$, which consists of $\mathrm{d} X / \mathrm{d} t$ by noncatalytic gasification and that by catalytic one. The noncatalytic gasification follows first-order kinetics with respect to the remaining fraction of unconverted char, i.e., $1-X$. On the other hand, the catalytic gasification obeys zeroth-order kinetics with respect to $1-X$.

$\frac{d X}{d t}=k_{\mathrm{nc}}(1-X)+\sum_{n} k_{\mathrm{C} n}$

The rate constants for the noncatalytic and catalytic gasification are denoted as $k_{\mathrm{nc}}$ and $k_{\mathrm{Cn}}$, respectively, where $n$ is the catalytic component $\mathrm{C} n(n=1-4)$. The rate of catalytic reaction, $k_{\text {Cn }}$ that represent the activity of $\mathrm{C} n$ catalyst can be described as a function of the effective amount of catalyst $\left(m_{\mathrm{Cn}}\right)$ and rate constant $\left(k^{\prime} \mathrm{C}\right)$.

$k_{\mathrm{C} n}=\frac{d X_{\mathrm{C} n}}{d t}=k^{\prime}{ }_{\mathrm{C}} m_{\mathrm{C} n}$

$k_{\mathrm{C} n, 0}=k_{\mathrm{C}}^{\prime} m_{\mathrm{C} n, 0}($ at $t=0)$

The authors realize that the rate of catalytic gasification is determined by the amount of catalyst retained in each char particle, $k_{\mathrm{Cn}}$ instead of the concentration of catalyst (i.e., its amount per mass or volume of char). While $k^{\prime}{ }_{\mathrm{C}}$ is a rate constant that defined to be common among the catalysts $1-4$. The activity of a catalyst, $k_{\mathrm{Cn}}$ changes along with the progress of char conversion due to the change of $m_{\mathrm{Cn}}$. Catalyst deactivation has responsible for the decreasing of $k_{\mathrm{Cn}}$ which commonly occurred in the gasification of lignite. During pyrolysis, the catalyst was dispersed in the char matrix on the atomic scale or in the form of nano-size particles, it experiences deactivation by agglomeration/growth mechanism. It increases the particle size of catalyst, decreases the catalyst activity per mass, and then $m_{\mathrm{Cn}}$. The reaction of the catalyst with mineral matter, such as oxides, silica, alumina, and aluminosilicate is the other important mechanism of deactivation. For both mechanisms above, the deactivation rate is a function of the concentration of the catalyst in the char matrix rather than the amount. Volatilization can also change $m_{\mathrm{Cn}}$, but it was not significant under the present gasification condition due to the absence of gas flow forced to pass through the char particles.

There is no urgency to measure or determine the absolute value of catalyst concentration, but it is necessary to express dependency of the concentration on $X$. A simple equation can evaluate the catalyst concentration for the gasification-induced enrichment of catalysts.

$C_{\mathrm{Cn}}=\frac{m_{\mathrm{C} n}}{1-X}$

Then, the rate of catalyst deactivation is assumed following the first-order kinetics concerning the catalyst concentration and is expressed by:

$\frac{d m_{\mathrm{C} n}}{d t}=k_{\text {loss }-\mathrm{n}} C_{\mathrm{C} n}=k_{\text {loss }-\mathrm{n}} \frac{m_{\mathrm{C} n}}{1-X}$

Transformation or activation of the catalyst precursor is a factor that can increase $m_{\mathrm{Cn}}$. The 
present authors have been performed preliminary experiments and analysis, and the results showed the necessity of assuming the presence of at least a type of catalyst precursor for gasification of chars from raw and washed coals. The present model assumes expediently that a single type of precursor $C_{\mathrm{Clprec}}$ is transformed exclusively into the catalyst $\mathrm{C} 1$. Then, eq. $\mathrm{V}$ is extended to the following equations:

$\frac{d m_{\mathrm{C} 1}}{d t}=k_{\mathrm{C} 1 \mathrm{prec}} C_{\mathrm{C} 1 \mathrm{prec}}-k_{\mathrm{loss}-1} C_{\mathrm{C} 1} n=1$

$\frac{d m_{\mathrm{C} n}}{d t}=-k_{\text {loss }-\mathrm{n}} C_{\mathrm{C} n} n \geq 2$

The concentration and the kinetics of the transformation of $C_{\mathrm{Clprec}}$ are defined the same way as a $C_{\mathrm{C} 1}$ in eqs.IV and $\mathrm{V}$, respectively. The kinetic model defines that the total amount of the catalysts and the catalyst precursor is unity at the beginning of gasification.

$\sum_{n} m_{\mathrm{C} n, 0}+m_{\mathrm{C} 1 \mathrm{prec}, 0}=1$

The rate of catalytic gasification at the initial point, $t=0$ is defined as ICA-1. ICA-1 represents the initial catalytic activity.

ICA-1 $=\sum_{n} k_{\mathrm{C} n, 0}=\sum_{n} k_{\mathrm{C} n}^{\prime} m_{\mathrm{C} n, 0}$

When the catalyst precursor, $C_{\mathrm{Cl} \text { prec}}$, presences in the char, it is also reasonable to define an initial and potential catalytic activity by considering $C_{\mathrm{Clprec}}$. Then, eq.IX is extended to eq.X.

ICA-2 $=k^{\prime}{ }_{\mathrm{C}} m_{\mathrm{C} 1 \text { prec }, 0}+\sum_{n} k_{\mathrm{C} n, 0}=k_{\mathrm{C}}^{\prime}\left(m_{\mathrm{C} 1 \text { prec }, 0}+\sum_{n} m_{\mathrm{C} n, 0}\right)=k_{\mathrm{C}}^{\prime}$

The catalytic activities, which described in eqs.IX and X, are hereafter called ICA-1 and ICA2 , respectively. Both are identical to each other when $m_{\mathrm{Clprec}}=0$. Meanwhile, the kinetics of catalyst deactivation is evaluated as a similar way to ICA:

ICD-1 $=\sum_{n} k_{\text {loss-n,0 }} C_{\mathrm{C} n, 0}$

ICD-2 $=k_{\text {loss-1,0 }}\left(C_{\mathrm{C} 1,0}+C_{\mathrm{C} 1 \text { prec }, 0}\right)+\sum_{n} k_{\text {loss-n }, 0} C_{\mathrm{C} n, 0}$ 


\section{SI.5. Concentration of metallic species in lignites and chars}

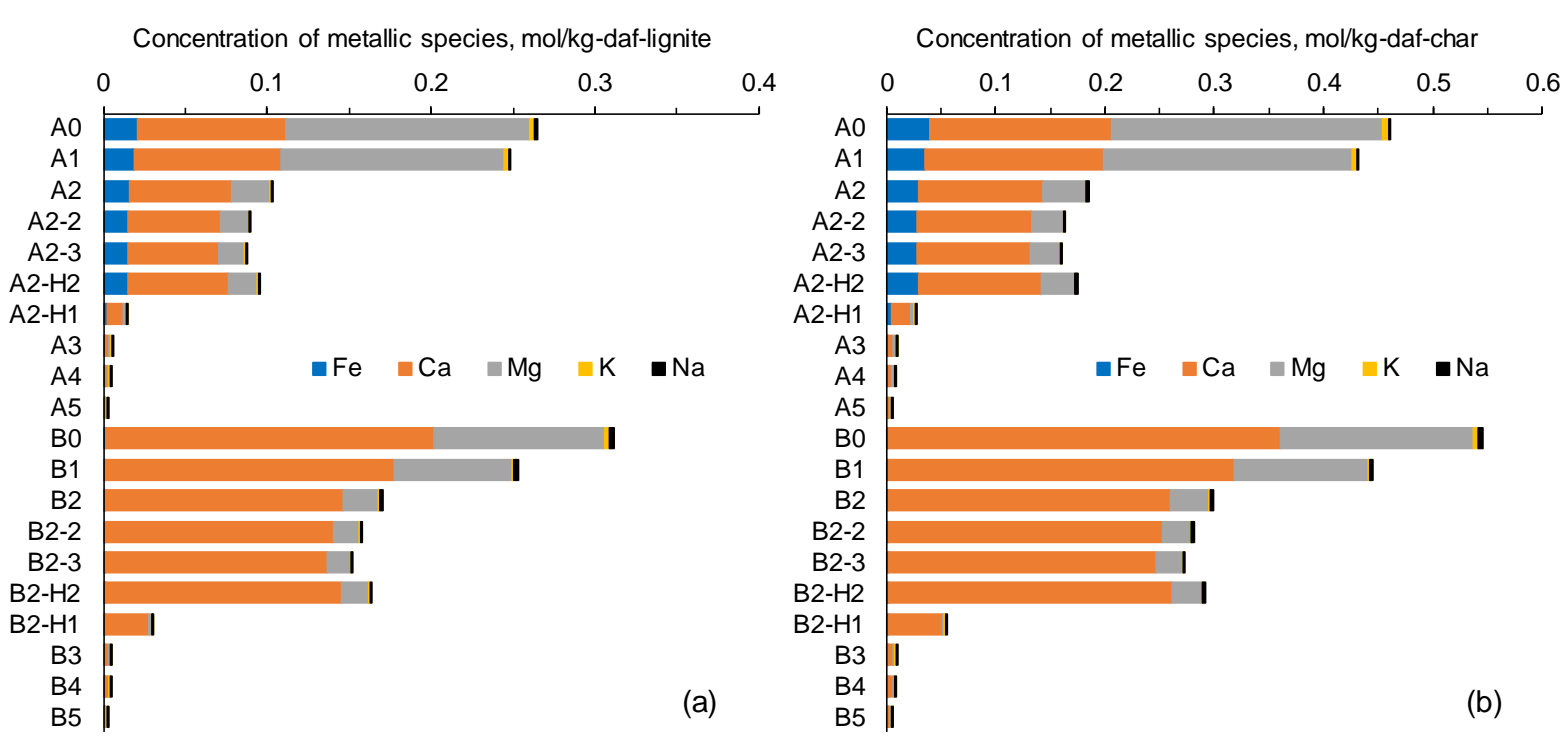

Figure SI.5. Concentrations of metallic species in (a) the lignites and (b) the chars. Concentrations of metallic species in the chars were calculated by considering the initial contents of metallic species in the lignites, the char yields, and the volatilization rates. The average volatilization rates of $\mathrm{K}$ and $\mathrm{Mg}$ for both $\mathrm{A} 0$ and $\mathrm{B} 0$ after the pyrolysis were $10 \%$ and $7 \%$, respectively. It was difficult to determine the volatilization rate of $\mathrm{Na}$ due to its low initial concentration, nevertheless a large amount of lignite ( $c a .100 \mathrm{mg}$ ) was used for the pyrolysis and quantification of AAEM species. Thus, the volatilization rate of $\mathrm{Na}$ was assumed to be equivalent to $\mathrm{K}$. It was safely concluded that volatilization of $\mathrm{Ca}$ did not occur during the pyrolysis as well gasification. For Fe, though not analyzed, it was safely assumed that there was no volatilization. 
SI.6. Lists of optimized kinetic parameters

\begin{tabular}{|c|c|c|c|c|c|}
\hline Sample ID & $\mathrm{A} 0$ & A1 & $\mathrm{A} 2$ & A2-2 & A2-3 \\
\hline$k_{\mathrm{ncg}} \min ^{-1}$ & $3.5 \times 10^{-3}$ & $3.5 \times 10^{-3}$ & $3.5 \times 10^{-3}$ & $3.5 \times 10^{-3}$ & $3.5 \times 10^{-3}$ \\
\hline ICA- $1, \min ^{-1}$ & $3.6 \times 10^{-1}$ & $3.3 \times 10^{-1}$ & $3.0 \times 10^{-1}$ & $2.3 \times 10^{-1}$ & $2.3 \times 10^{-1}$ \\
\hline ICA-2, $\min ^{-1}$ & $3.6 \times 10^{-1}$ & $3.3 \times 10^{-1}$ & $3.1 \times 10^{-1}$ & $2.6 \times 10^{-1}$ & $2.5 \times 10^{-1}$ \\
\hline ICD-1, $\min ^{-1}$ & $2.5 \times 10^{-2}$ & $2.1 \times 10^{-1}$ & $2.0 \times 10^{-1}$ & $1.1 \times 10^{-1}$ & $1.1 \times 10^{-1}$ \\
\hline ICD-2, $\min ^{-1}$ & $2.5 \times 10^{-1}$ & $2.1 \times 10^{-1}$ & $2.1 \times 10^{-1}$ & $1.3 \times 10^{-1}$ & $1.3 \times 10^{-1}$ \\
\hline$C_{\text {C1prec }},-$ & $4.5 \times 10^{-3}$ & $2.2 \times 10^{-2}$ & $5.0 \times 10^{-2}$ & $1.0 \times 10^{-1}$ & $9.0 \times 10^{-2}$ \\
\hline$C_{\mathrm{C} 1,0},-$ & $5.4 \times 10^{-1}$ & $5.8 \times 10^{-1}$ & $6.7 \times 10^{-1}$ & $4.3 \times 10^{-1}$ & $4.3 \times 10^{-1}$ \\
\hline$C_{\mathrm{C} 2,0},-$ & $3.1 \times 10^{-1}$ & $2.4 \times 10^{-1}$ & $1.7 \times 10^{-1}$ & $3.8 \times 10^{-1}$ & $3.9 \times 10^{-1}$ \\
\hline$C_{\mathrm{C} 3,0},-$ & $1.3 \times 10^{-1}$ & $1.5 \times 10^{-1}$ & $8.9 \times 10^{-2}$ & $9.1 \times 10^{-2}$ & $9.4 \times 10^{-2}$ \\
\hline$C_{\mathrm{C} 4,0},-$ & $2.1 \times 10^{-2}$ & $1.2 \times 10^{-2}$ & $2.0 \times 10^{-2}$ & $1.1 \times 10^{-3}$ & $7.0 \times 10^{-4}$ \\
\hline$k_{\text {Clprec }}, \min ^{-1}$ & $1.2 \times 10^{-2}$ & $6.0 \times 10^{-1}$ & 2.00 & 2.10 & 2.10 \\
\hline$k_{\mathrm{Cl}, 0}, \min ^{-1}$ & $1.9 \times 10^{-1}$ & $1.9 \times 10^{-1}$ & $2.1 \times 10^{-1}$ & $1.1 \times 10^{-1}$ & $1.1 \times 10^{-1}$ \\
\hline$k_{\mathrm{C} 2,0}, \min ^{-1}$ & $1.1 \times 10^{-1}$ & $7.9 \times 10^{-2}$ & $5.3 \times 10^{-2}$ & $9.9 \times 10^{-2}$ & $9.9 \times 10^{-2}$ \\
\hline$k_{\mathrm{C} 3,0}, \min ^{-1}$ & $4.5 \times 10^{-2}$ & $5.1 \times 10^{-2}$ & $2.8 \times 10^{-2}$ & $2.4 \times 10^{-2}$ & $2.4 \times 10^{-2}$ \\
\hline$k_{\mathrm{C} 4,0}, \mathrm{~min}^{-1}$ & $7.5 \times 10^{-3}$ & $4.1 \times 10^{-3}$ & $6.3 \times 10^{-3}$ & $2.8 \times 10^{-4}$ & $1.8 \times 10^{-4}$ \\
\hline$k_{\text {loss-1 }}, \min ^{-1}$ & $3.8 \times 10^{-1}$ & $3.2 \times 10^{-1}$ & $2.9 \times 10^{-1}$ & $2.1 \times 10^{-1}$ & $2.2 \times 10^{-1}$ \\
\hline$k_{\text {loss-2 }}, \min ^{-1}$ & $1.3 \times 10^{-1}$ & $9.2 \times 10^{-2}$ & $3.7 \times 10^{-2}$ & $4.4 \times 10^{-2}$ & $4.7 \times 10^{-2}$ \\
\hline$k_{\text {loss-3}}, \min ^{-1}$ & $1.1 \times 10^{-2}$ & $1.2 \times 10^{-2}$ & $1.2 \times 10^{-2}$ & $5.8 \times 10^{-3}$ & $5.8 \times 10^{-3}$ \\
\hline$k_{\text {loss- } 4, \min ^{-1}}$ & $1.4 \times 10^{-4}$ & $2.0 \times 10^{-3}$ & $7.0 \times 10^{-4}$ & $1.0 \times 10^{-4}$ & $1.0 \times 10^{-4}$ \\
\hline Sample ID & $\mathrm{A} 2-\mathrm{H} 2$ & A2-H1 & $\mathrm{A} 3$ & A4 & A5 \\
\hline$k_{\text {ncg }} \min ^{-1}$ & $3.5 \times 10^{-3}$ & $3.5 \times 10^{-3}$ & $3.5 \times 10^{-3}$ & $3.5 \times 10^{-3}$ & $3.5 \times 10^{-3}$ \\
\hline ICA- $1, \min ^{-1}$ & $2.7 \times 10^{-1}$ & $1.1 \times 10^{-1}$ & $9.0 \times 10^{-4}$ & $4.3 \times 10^{-4}$ & \\
\hline ICA- $2, \min ^{-1}$ & $2.9 \times 10^{-1}$ & $1.2 \times 10^{-1}$ & $4.8 \times 10^{-3}$ & $2.5 \times 10^{-3}$ & \\
\hline ICD-1, $\min ^{-1}$ & $1.3 \times 10^{-1}$ & $4.0 \times 10^{-2}$ & $1.4 \times 10^{-4}$ & $2.4 \times 10^{-5}$ & \\
\hline ICD-2, $\min ^{-1}$ & $1.5 \times 10^{-1}$ & $4.4 \times 10^{-2}$ & $1.8 \times 10^{-3}$ & $7.5 \times 10^{-4}$ & \\
\hline$C_{\mathrm{Clprec}},-$ & $7.0 \times 10^{-2}$ & $7.5 \times 10^{-2}$ & $8.1 \times 10^{-1}$ & $8.3 \times 10^{-1}$ & \\
\hline$C_{\mathrm{C} 1,0},-$ & $5.3 \times 10^{-1}$ & $6.3 \times 10^{-1}$ & $6.0 \times 10^{-2}$ & & \\
\hline$C_{\mathrm{C} 2,0},-$ & $2.9 \times 10^{-1}$ & $2.0 \times 10^{-1}$ & $1.3 \times 10^{-1}$ & $1.8 \times 10^{-1}$ & \\
\hline$C_{\mathrm{C} 3,0},-$ & $1.1 \times 10^{-1}$ & $8.3 \times 10^{-2}$ & & & \\
\hline$C_{\mathrm{C} 4,0},-$ & $1.2 \times 10^{-3}$ & $8.5 \times 10^{-3}$ & & & \\
\hline$k_{\mathrm{Clprec}}, \min ^{-1}$ & 1.40 & 1.10 & $2.1 \times 10^{-2}$ & $2.4 \times 10^{-2}$ & \\
\hline$k_{\mathrm{Cl}, 0}, \min ^{-1}$ & $1.6 \times 10^{-1}$ & $7.7 \times 10^{-2}$ & $2.9 \times 10^{-4}$ & & \\
\hline$k_{\mathrm{C} 2,0}, \min ^{-1}$ & $8.5 \times 10^{-2}$ & $2.4 \times 10^{-2}$ & $6.2 \times 10^{-4}$ & $4.3 \times 10^{-4}$ & \\
\hline$k_{\mathrm{C} 3,0}, \min ^{-1}$ & $3.1 \times 10^{-2}$ & $1.0 \times 10^{-2}$ & & & \\
\hline$k_{\mathrm{C} 4,0}, \min ^{-1}$ & $3.5 \times 10^{-4}$ & $1.0 \times 10^{-3}$ & & & \\
\hline$k_{\text {loss-1 }}, \min ^{-1}$ & $2.2 \times 10^{-1}$ & $5.8 \times 10^{-2}$ & $2.1 \times 10^{-3}$ & $8.8 \times 10^{-4}$ & \\
\hline$k_{\text {loss-2 }}, \min ^{-1}$ & $4.9 \times 10^{-2}$ & $1.4 \times 10^{-2}$ & $1.5 \times 10^{-4}$ & $1.4 \times 10^{-4}$ & \\
\hline$k_{\text {loss- } 3}, \min ^{-1}$ & $7.7 \times 10^{-3}$ & $3.8 \times 10^{-3}$ & & & \\
\hline$k_{\text {loss- } 4}, \min ^{-1}$ & $1.0 \times 10^{-4}$ & $5.0 \times 10^{-5}$ & & & \\
\hline
\end{tabular}




\begin{tabular}{|c|c|c|c|c|c|}
\hline Sample ID & B0 & B1 & $\mathrm{B} 2$ & B2-2 & B2-3 \\
\hline$k_{\text {ncg }} \min ^{-1}$ & $3.5 \times 10^{-3}$ & $3.5 \times 10^{-3}$ & $3.5 \times 10^{-3}$ & $3.5 \times 10^{-3}$ & $3.5 \times 10^{-3}$ \\
\hline ICA- $1, \min ^{-1}$ & $4.8 \times 10^{-1}$ & $4.5 \times 10^{-1}$ & $3.8 \times 10^{-1}$ & $3.2 \times 10^{-1}$ & $3.1 \times 10^{-1}$ \\
\hline ICA- $2, \min ^{-1}$ & $4.8 \times 10^{-1}$ & $4.8 \times 10^{-1}$ & $4.2 \times 10^{-1}$ & $3.6 \times 10^{-1}$ & $3.4 \times 10^{-1}$ \\
\hline ICD- $1, \min ^{-1}$ & $2.3 \times 10^{-2}$ & $1.8 \times 10^{-1}$ & $1.4 \times 10^{-1}$ & $9.2 \times 10^{-2}$ & $9.8 \times 10^{-2}$ \\
\hline ICD-2, $\min ^{-1}$ & $2.3 \times 10^{-1}$ & $1.9 \times 10^{-1}$ & $1.5 \times 10^{-1}$ & $1.0 \times 10^{-1}$ & $1.1 \times 10^{-1}$ \\
\hline$C_{\text {Clprec }},-$ & $6.0 \times 10^{-3}$ & $6.4 \times 10^{-2}$ & $8.0 \times 10^{-2}$ & $1.0 \times 10^{-1}$ & $9.2 \times 10^{-2}$ \\
\hline$C_{\mathrm{C} 1,0},-$ & $6.6 \times 10^{-1}$ & $7.5 \times 10^{-1}$ & $6.9 \times 10^{-1}$ & $7.8 \times 10^{-1}$ & $7.5 \times 10^{-1}$ \\
\hline$C_{\mathrm{C} 2,0},-$ & $2.9 \times 10^{-1}$ & $1.3 \times 10^{-1}$ & $1.4 \times 10^{-1}$ & $6.5 \times 10^{-2}$ & $8.5 \times 10^{-2}$ \\
\hline$C_{\mathrm{C} 3,0},-$ & $3.7 \times 10^{-2}$ & $5.5 \times 10^{-2}$ & $8.3 \times 10^{-2}$ & $5.1 \times 10^{-2}$ & $6.7 \times 10^{-2}$ \\
\hline$C_{\mathrm{C} 4,0},-$ & $9.1 \times 10^{-3}$ & $3.0 \times 10^{-3}$ & $1.0 \times 10^{-2}$ & $9.0 \times 10^{-4}$ & $5.8 \times 10^{-3}$ \\
\hline$k_{\mathrm{Clprec}}, \min ^{-1}$ & $7.0 \times 10^{-1}$ & 2.00 & 1.90 & $8.0 \times 10^{-1}$ & 1.00 \\
\hline$k_{\mathrm{C} 1,0}, \min ^{-1}$ & $3.2 \times 10^{-1}$ & $3.6 \times 10^{-1}$ & $2.9 \times 10^{-1}$ & $2.8 \times 10^{-1}$ & $2.6 \times 10^{-1}$ \\
\hline$k_{\mathrm{C} 2,0}, \min ^{-1}$ & $1.4 \times 10^{-1}$ & $6.3 \times 10^{-2}$ & $5.8 \times 10^{-2}$ & $2.3 \times 10^{-2}$ & $2.9 \times 10^{-2}$ \\
\hline$k_{\mathrm{C} 3,0}, \min ^{-1}$ & $1.8 \times 10^{-2}$ & $2.6 \times 10^{-2}$ & $3.5 \times 10^{-2}$ & $1.8 \times 10^{-2}$ & $2.3 \times 10^{-2}$ \\
\hline$k_{\mathrm{C} 4,0}, \min ^{-1}$ & $4.4 \times 10^{-3}$ & $1.4 \times 10^{-3}$ & $4.2 \times 10^{-4}$ & $3.2 \times 10^{-4}$ & $2.0 \times 10^{-3}$ \\
\hline$k_{\text {loss-1 }}, \min ^{-1}$ & $3.3 \times 10^{-1}$ & $2.3 \times 10^{-1}$ & $1.9 \times 10^{-1}$ & $1.2 \times 10^{-1}$ & $1.3 \times 10^{-1}$ \\
\hline$k_{\text {loss-2 }}, \min ^{-1}$ & $5.3 \times 10^{-2}$ & $4.0 \times 10^{-2}$ & $5.9 \times 10^{-2}$ & $1.8 \times 10^{-2}$ & $2.9 \times 10^{-2}$ \\
\hline$k_{\text {loss-3}}, \min ^{-1}$ & $5.1 \times 10^{-3}$ & $8.0 \times 10^{-3}$ & $1.2 \times 10^{-2}$ & $6.4 \times 10^{-3}$ & $9.2 \times 10^{-3}$ \\
\hline$k_{\text {loss-4 }}, \min ^{-1}$ & $5.6 \times 10^{-3}$ & $7.0 \times 10^{-3}$ & $2.0 \times 10^{-3}$ & $2.0 \times 10^{-2}$ & $1.0 \times 10^{-5}$ \\
\hline Sample ID & B2-H2 & B2-H1 & B3 & B4 & B5 \\
\hline$k_{\text {ncg, }} \min ^{-1}$ & $3.5 \times 10^{-3}$ & $3.5 \times 10^{-3}$ & $3.5 \times 10^{-3}$ & $3.5 \times 10^{-3}$ & $3.5 \times 10^{-3}$ \\
\hline ICA- $1, \min ^{-1}$ & $3.6 \times 10^{-1}$ & $1.4 \times 10^{-1}$ & $9.1 \times 10^{-4}$ & $1.6 \times 10^{-4}$ & \\
\hline ICA-2, $\min ^{-1}$ & $3.9 \times 10^{-1}$ & $1.6 \times 10^{-1}$ & $4.6 \times 10^{-3}$ & $1.9 \times 10^{-3}$ & \\
\hline ICD-1, $\min ^{-1}$ & $1.2 \times 10^{-1}$ & $5.0 \times 10^{-2}$ & $2.4 \times 10^{-4}$ & $4.2 \times 10^{-8}$ & \\
\hline ICD-2, $\min ^{-1}$ & $1.3 \times 10^{-1}$ & $6.0 \times 10^{-2}$ & $1.7 \times 10^{-3}$ & $5.3 \times 10^{-4}$ & \\
\hline$C_{\text {Clprec }},-$ & $8.0 \times 10^{-2}$ & $1.4 \times 10^{-1}$ & $8.0 \times 10^{-1}$ & $9.2 \times 10^{-1}$ & \\
\hline$C_{\mathrm{C} 1,0},-$ & $6.8 \times 10^{-1}$ & $6.6 \times 10^{-1}$ & $1.2 \times 10^{-1}$ & & \\
\hline$C_{\mathrm{C} 2,0},-$ & $1.8 \times 10^{-2}$ & $1.2 \times 10^{-1}$ & $7.8 \times 10^{-2}$ & $8.4 \times 10^{-2}$ & \\
\hline$C_{\mathrm{C} 3,0},-$ & $5.3 \times 10^{-2}$ & $7.4 \times 10^{-2}$ & & & \\
\hline$C_{\mathrm{C} 4,0},-$ & $5.6 \times 10^{-3}$ & $9.7 \times 10^{-3}$ & & & \\
\hline$k_{\text {Clprec }}, \min ^{-1}$ & 2.00 & 1.00 & $1.3 \times 10^{-2}$ & $1.2 \times 10^{-1}$ & \\
\hline$k_{\mathrm{C} 1,0}, \min ^{-1}$ & $2.7 \times 10^{-1}$ & $1.1 \times 10^{-1}$ & $5.6 \times 10^{-4}$ & & \\
\hline$k_{\mathrm{C} 2,0}, \min ^{-1}$ & $7.0 \times 10^{-2}$ & $1.9 \times 10^{-2}$ & $3.5 \times 10^{-4}$ & $1.6 \times 10^{-4}$ & \\
\hline$k_{\mathrm{C} 3,0}, \min ^{-1}$ & $2.1 \times 10^{-2}$ & $1.2 \times 10^{-2}$ & & & \\
\hline$k_{\mathrm{C} 4,0}, \min ^{-1}$ & $2.2 \times 10^{-3}$ & $1.5 \times 10^{-3}$ & & & \\
\hline$k_{\text {loss-1 }}, \min ^{-1}$ & $1.6 \times 10^{-1}$ & $7.2 \times 10^{-2}$ & $1.9 \times 10^{-3}$ & $5.8 \times 10^{-4}$ & \\
\hline$k_{\text {loss-2 }}, \min ^{-1}$ & $4.0 \times 10^{-2}$ & $2.4 \times 10^{-2}$ & $7.8 \times 10^{-5}$ & $5.0 \times 10^{-7}$ & \\
\hline$k_{\text {loss-3}}, \min ^{-1}$ & $1.1 \times 10^{-2}$ & $4.0 \times 10^{-3}$ & & & \\
\hline$k_{\text {loss- } 4}, \min ^{-1}$ & $1.0 \times 10^{-5}$ & $6.0 \times 10^{-4}$ & & & \\
\hline
\end{tabular}




\section{SI.7. $\mathrm{d} X / \mathrm{d} t$ vs $X$ profiles for the individual chars}
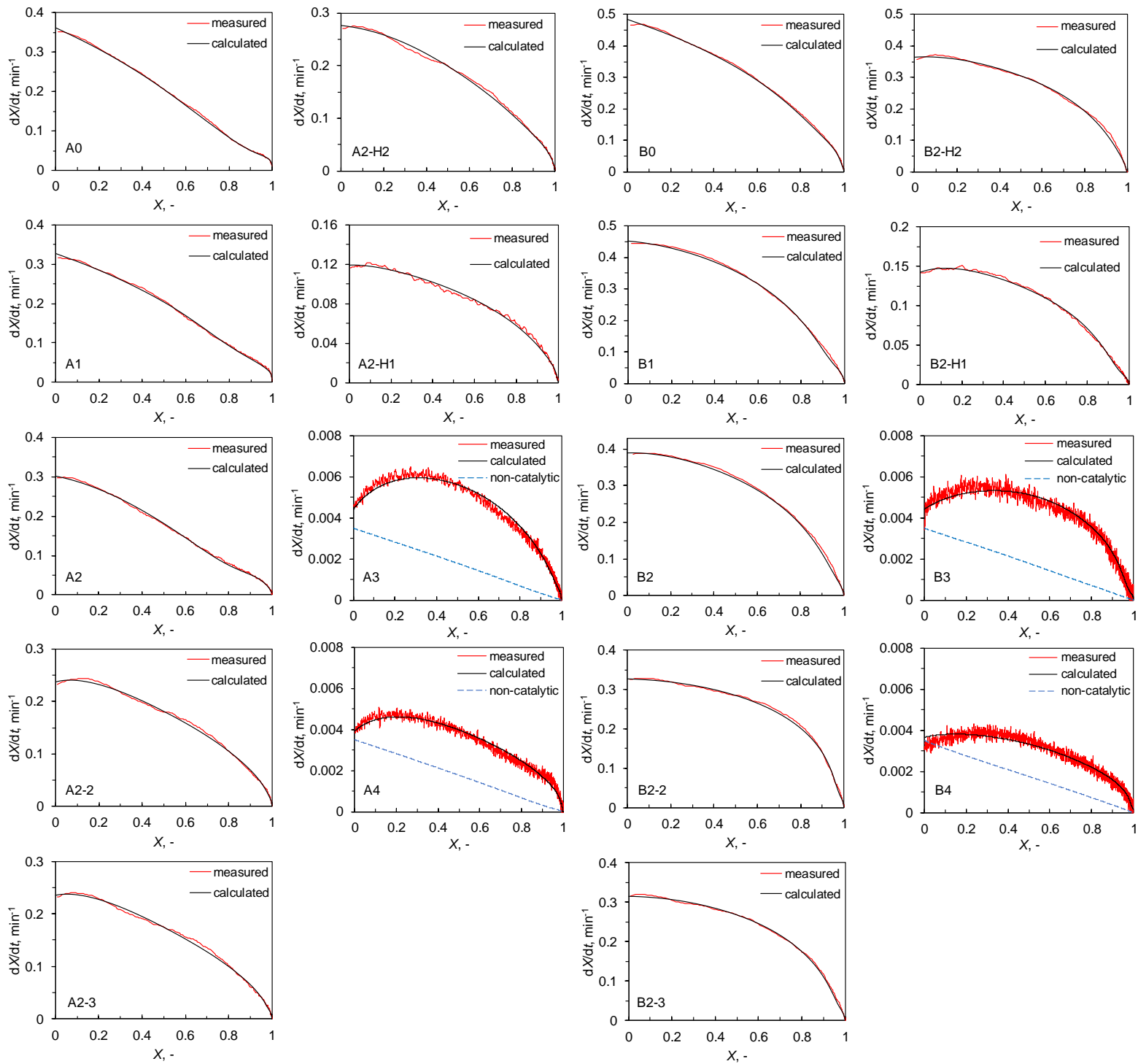

Figure SI.7. Measured and calculated changes in $\mathrm{d} X / \mathrm{dt}$ with $X$ for gasification of chars from original and washed lignites. The blue-colored broken lines were drawn for A3, A4, B3 and B4 to show $\mathrm{d} X / \mathrm{d} t$ for the noncatalytic gasification with $k_{\mathrm{nc}}=0.0035 \mathrm{~min}^{-1}$. For many of the chars, $\mathrm{d} X / \mathrm{d} t$ changes through a maximum. This is interpreted by the model that the catalyst precursor (C1prec) is transformed into the $\mathrm{C} 1$ catalyst. 


\section{SI.8. Gibbs free energy for reactions involving catalytic species}

\begin{tabular}{|c|c|c|c|c|}
\hline No. & Reaction & $\begin{array}{c}\Delta G_{\mathrm{r}} \text { at } 900^{\circ} \mathrm{C}, \\
\mathrm{kJ} / \text { mol-metal }\end{array}$ & $K_{\mathrm{p}}$ & Remark \\
\hline 1 & $\mathrm{Na}_{2} \mathrm{CO}_{3}+\mathrm{C}=\mathrm{Na}_{2} \mathrm{O}+2 \mathrm{Cl}$ & 109 & $1.5 \times 10^{-5}$ & $\mathrm{Na}_{2} \mathrm{CO}_{3}$ is the major species. Overall \\
\hline 2 & $\mathrm{Na}_{2} \mathrm{O}+\mathrm{CO}_{2}=\mathrm{Na}_{2} \mathrm{CO}_{3}$ & -142 & $2.2 \times 10^{6}$ & $\Delta G$ for $\mathrm{R} 1$ and $\mathrm{R} 2$ is negative, and \\
\hline 3 & $\mathrm{Na}_{2} \mathrm{O}+\mathrm{C}=2 \mathrm{Na}+\mathrm{CO}$ & 46 & $8.8 \times 10^{-3}$ & carbonate-oxide cycles are possible. \\
\hline 4 & $2 \mathrm{Na}+\mathrm{CO}_{2}=\mathrm{Na}_{2} \mathrm{O}+\mathrm{CO}$ & -80 & $3.7 \times 10^{3}$ & but formation of $\mathrm{Na}_{2} \mathrm{O}$ is difficult. \\
\hline 5 & $\mathrm{~K}_{2} \mathrm{CO}_{3}+\mathrm{C}=\mathrm{K}_{2} \mathrm{O}+2 \mathrm{CO}$ & 181 & $8.6 \times 10^{-9}$ & \multirow{4}{*}{$\begin{array}{l}\mathrm{K}_{2} \mathrm{CO}_{3} \text { is the major species. Overall } \\
\Delta G \text { for } \mathrm{R} 5 \text { and } \mathrm{R} 6 \text { is negative, and } \\
\text { carbonate-oxide cycles are possible. } \\
\text { Metal-oxide cycles are possible, too, } \\
\text { but formation of } \mathrm{K}_{2} \mathrm{O} \text { is difficult. }\end{array}$} \\
\hline 6 & $\mathrm{~K}_{2} \mathrm{O}+\mathrm{CO}_{2}=\mathrm{K}_{2} \mathrm{CO}_{3}$ & -215 & $3.7 \times 10^{9}$ & \\
\hline 7 & $\mathrm{~K}_{2} \mathrm{O}+\mathrm{C}=2 \mathrm{~K}+\mathrm{CO}$ & -13 & 4.0 & \\
\hline 8 & $2 \mathrm{~K}+\mathrm{CO}_{2}=\mathrm{K}_{2} \mathrm{O}+\mathrm{CO}$ & -20 & 8.1 & \\
\hline 9 & $\mathrm{MgCO}_{3}+\mathrm{C}=\mathrm{MgO}+2 \mathrm{CO}$ & -139 & $1.5 \times 10^{6}$ & \multirow{3}{*}{$\begin{array}{l}\mathrm{MgO} \text { is the major species. } \mathrm{MgCO}_{3} \text { is } \\
\text { reactive with } \mathrm{C} \text {, but its presence is } \\
\text { difficult due to that of } \mathrm{R} 10 \text {. Then, the } \\
\text { catalysis of } \mathrm{MgCO}_{3} \text { is implausible. }\end{array}$} \\
\hline 10 & $\mathrm{MgO}+\mathrm{CO}_{2}=\mathrm{MgCO}_{3}$ & 105 & $2.2 \times 10^{-5}$ & \\
\hline 11 & $\mathrm{MgO}+\mathrm{C}=\mathrm{Mg}+\mathrm{CO}$ & 259 & $2.9 \times 10^{-12}$ & \\
\hline 12 & $\mathrm{CaCO}_{3}+\mathrm{C}=\mathrm{CaO}+2 \mathrm{CO}$ & -43 & $7.9 \times 10^{1}$ & \multirow{3}{*}{$\begin{array}{l}\text { Major species, } \mathrm{CaO} \text { and } \mathrm{CaCO}_{3} \\
\text { participate in carbonate-oxide catalytic } \\
\text { cycles. Formation of metallic } \mathrm{Ca} \text { is } \\
\text { difficult. }\end{array}$} \\
\hline 13 & $\mathrm{CaO}+\mathrm{CO}_{2}=\mathrm{CaCO}_{3}$ & 9 & $4.1 \times 10^{-1}$ & \\
\hline 14 & $\mathrm{CaO}+\mathrm{C}=\mathrm{Ca}+\mathrm{CO}$ & 295 & $7.3 \times 10^{-14}$ & \\
\hline 15 & $\mathrm{Fe}_{3} \mathrm{O}_{4}+\mathrm{C}=3 \mathrm{FeO}+\mathrm{CO}$ & -73 & $1.8 \times 10^{3}$ & \multirow{6}{*}{$\begin{array}{l}\text { Integration of } K_{\mathrm{p}} \text { of } \mathrm{R} 15-\mathrm{R} 20 \text { leads to } \\
\text { abundances of } \mathrm{FeO} \text { and } \mathrm{Fe}_{3} \mathrm{O}_{4} \text {, and less } \\
\text { abundance of } \mathrm{Fe} . \mathrm{FeO}_{-}-\mathrm{Fe}_{3} \mathrm{O}_{4} \text { cycles } \\
\text { are major catalytic cycles. }\end{array}$} \\
\hline 16 & $3 \mathrm{FeO}+\mathrm{CO}_{2}=\mathrm{Fe}_{3} \mathrm{O}_{4}+\mathrm{CO}$ & 39 & $1.8 \times 10^{-2}$ & \\
\hline 17 & $\mathrm{FeO}+\mathrm{CO}=\mathrm{Fe}+\mathrm{CO}_{2}$ & 9 & $4.1 \times 10^{-1}$ & \\
\hline 18 & $\mathrm{FeO}+\mathrm{C}=\mathrm{Fe}+\mathrm{CO}$ & -25 & $1.3 \times 10^{1}$ & \\
\hline 19 & $\mathrm{FeO}+\mathrm{CO}_{2}=\mathrm{FeO}+\mathrm{CO}$ & -9 & 2.4 & \\
\hline 20 & $\mathrm{FeO}+\mathrm{CO}_{2}=\mathrm{FeCO}_{3}$ & 137 & $7.7 \times 10^{-7}$ & \\
\hline
\end{tabular}


SI.9. Inorganic compounds in char and ash detected by XRD

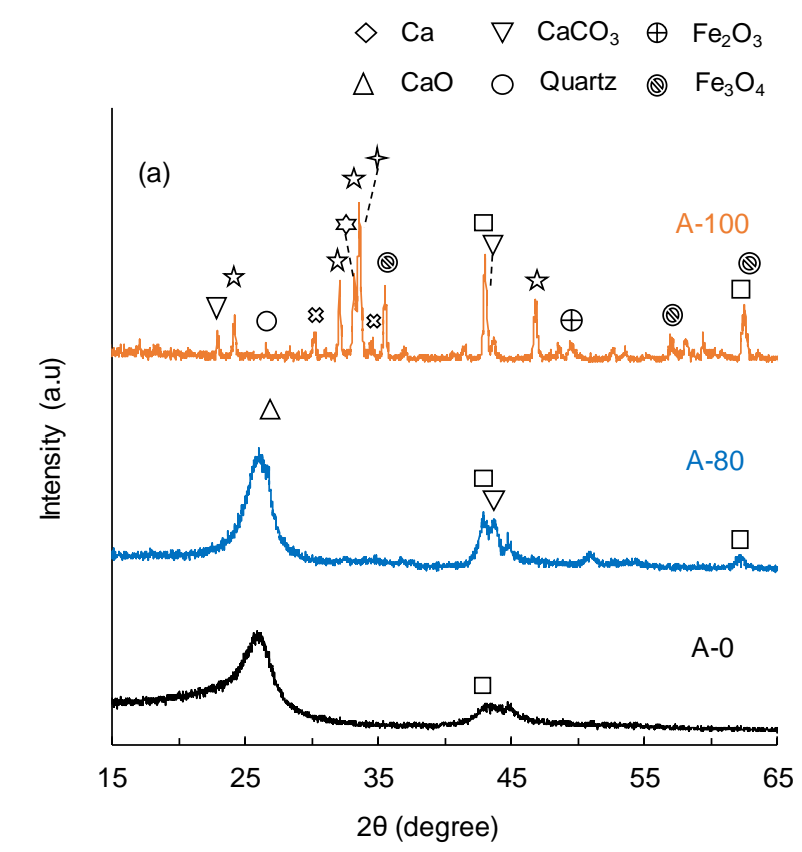

$\square \mathrm{MgO} \& \mathrm{CaFe}_{2} \mathrm{O}_{4} \& \mathrm{Ca}_{2} \mathrm{Fe}_{2} \mathrm{MgO}_{6}$

$\triangle \mathrm{CaO} O$ Quartz \& $\mathrm{Fe}_{3} \mathrm{O}_{4}$ is $\mathrm{Ca}_{2} \mathrm{Fe}_{2} \mathrm{O}_{5}$ \& $\mathrm{CaMgSiO}_{4}$

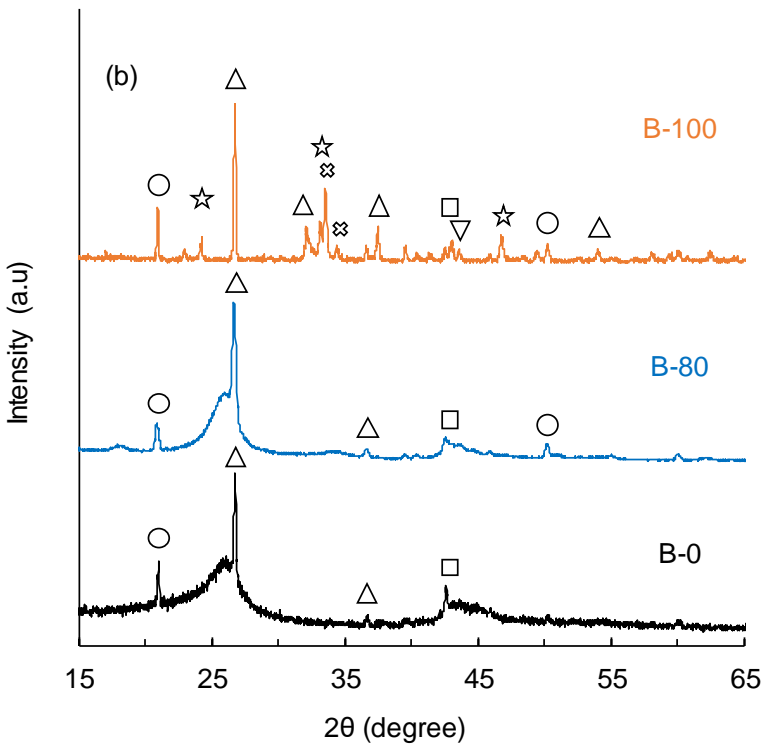

Figure SI.9. XRD patterns of the chars at $X=0,80 \%$ and $100 \%$ from (a) A0 and (b) B0 lignites. 